\title{
Non-radial pulsations in the $\gamma$ Doradus star HD 195068
}

\author{
S. Jankov ${ }^{1,2,3}$, P. Mathias ${ }^{1}$, E. Chapellier ${ }^{1}$, J.-M. Le Contel $^{1}$, and J.-P. Sareyan ${ }^{1}$ \\ 1 Observatoire de la Côte d'Azur, Dpt. Gemini, UMR 6203, BP 4229, 06304 Nice Cedex 04, France \\ e-mail: Slobodan. Jankov@obs-azur.fr \\ 2 Laboratoire Univ. d'Astroph. de Nice (LUAN), UMR 6525, Parc Valrose, 06108 Nice Cedex 02, France \\ 3 Astronomical Observatory, MSEP of Serbia 146007, Volgina 7, 11050 Belgrade, Serbia and Montenegro
}

Received 22 March 2005 / Accepted 31 January 2006

\section{ABSTRACT}

\begin{abstract}
We present high resolution spectroscopic observations of the $\gamma$ Doradus star HD 195068. About 230 spectra were collected over 2 years. Time series analysis performed on radial velocity data shows a main peak at $1.61 \mathrm{~d}^{-1}$, a frequency not yet detected in photometry. The Hipparcos photometric $1.25 \mathrm{~d}^{-1}$ frequency is easily recovered as is $1.30 \mathrm{~d}^{-1}$ while the third photometric frequency, $0.97 \mathrm{~d}^{-1}$, is only marginally present. The good quality of our data, which includes 196 spectra collected over seven consecutive nights, shows that both the $1.61 \mathrm{~d}^{-1}$ and intermediate $1.27 \mathrm{~d}^{-1}$ (mixture of 1.25 and $1.30 \mathrm{~d}^{-1}$ ) frequencies are present in the line profile variations. Using the Fourier-Doppler Imaging (FDI) method, the variability associated with $1.61 \mathrm{~d}^{-1}$ can be successfully modeled by a non-radial pulsation mode $\ell=5 \pm 1,|m|=4 \pm 1$. For the intermediate frequency $1.27 \mathrm{~d}^{-1}$ we deduce $\ell=4 \pm 1,|m|=3 \pm 1$. Evidence that the star is not pulsating in the radial mode $(\ell=0)$ rules out a previous classification as an RR Lyrae type star. We investigate the time variability of FDI power spectra concluding that the observed temporal variability of modes can be explained by a beating phenomenon between closely spaced frequencies of two non-radial modes. The distribution of the oscillation power within the line profile indicates that there is a significant tangential velocity component of oscillations characteristic of high radial order gravity modes which are predicted to be observed in $\gamma$ Doradus type stars.
\end{abstract}

Key words. line: profiles - stars: variables: general - stars: oscillations

\section{Introduction}

The $\gamma$ Doradus stars are a recently discovered class of periodically variable stars with time scales of 0.4 to 3 days. These brightness variations are presumably due to non-radial gravitymode pulsators of high radial overtones. Kaye et al. (1999a) have given an accurate description of the $\gamma$ Doradus variables. In particular, they pointed out that such pulsators can easily be confused with other variables whose light modulations are induced by $p$-mode pulsations, rotation or binarity. The destabilizing mechanism is probably due to the flux modulation induced by the upper convective layer (Guzik et al. 2000; Dupret et al. 2004; Grigahcène 2004).

Only some of these stars have been studied in detail: by means of time-resolved line profile analysis and by multisite photometric campaigns. Mathias et al. (2004a, Paper I) presented the first results of a 2-year high-resolution spectroscopy campaign of $59 \gamma$ Doradus candidates, most of which were discovered using the Hipparcos astrometric mission (ESA 1997). In the present study we concentrate on the star HD $195068=$ HIP $100859=$ V212 1 Cyg $=$ HR $7828(V=5.736$, F0V) since its prominent line profile variations (Paper I) should allow us to study their origin and nature. The light variability of HD 195068 was discovered by the Hipparcos mission (ESA 1997) and was suggested to belong to the class of RR Lyrae, in addition to presenting a strong Blazkho effect (Kazarovets et al. 1999). However, Eyer (1998) identified it as a possible $\gamma$ Doradus variable. Using Hipparcos data, Handler (1999) derived a $1.25 \mathrm{~d}^{-1}$ frequency, and a possible second one at

* Based on observations obtained at the Observatoire de HauteProvence.
$0.270 \mathrm{~d}^{-1}$. As a result of this, he classified the star as a prime $\gamma$ Doradus candidate. Using high resolution spectroscopy, Fekel et al. (2003) detected line asymmetries and favored the line profile variations (LPV) interpretation rather than the spectroscopic binary hypothesis. From precise photometric observations, Henry et al. (2005) detected 3 frequencies including $1.2507 \mathrm{~d}^{-1}, 1.2986 \mathrm{~d}^{-1}$ and $0.9652 \mathrm{~d}^{-1}$, and definitely classified the star as a $\gamma$ Doradus. The projected rotation velocity is $V_{\mathrm{e}} \sin i=46 \mathrm{~km} \mathrm{~s}^{-1}$ (Paper I).

The present paper concerns time series analysis and mode identification for HD 195068 using homogeneous spectroscopy. We present the results of an analysis of the behavior of selected photospheric lines observed during an additional campaign devoted to HD 195068. The observations and data reduction are described in Sect. 2, while Sect. 3 deals with the frequency analysis of the radial velocities and line profile variations. The non-radial behavior of the star is studied in Sect. 4 using Doppler Mapping and the two-dimensional Fourier-Doppler Imaging method for line-profile analysis. The advent of these methods has allowed us to detect and identify the pulsation modes described below. We discuss our results in Sect. 5, and give concluding remarks in Sect. 6.

\section{Observations and data reduction}

The spectroscopic campaign was spread over 2 years, from August 2001 to August 2003. However, most of our data cover the period July 28 to August 4, 2003, and were made at the Observatoire de Haute-Provence using the AURELIE spectrograph attached to the $1.52 \mathrm{~m}$ telescope. The spectral domain covers 4470-4540 $\AA$ with a resolving power $\lambda / \Delta \lambda=55000$. 
Table 1. Log of observations. The second column gives the beginning and the end of each night with respect to the reference epoch HJD $=2452100$ and the third the number of spectra $N$.

\begin{tabular}{|c|c|c|}
\hline Night & Range [d] & $\bar{N}$ \\
\hline 1 & $36.34232-36.35773$ & 2 \\
\hline 2 & 49.47244 & 1 \\
\hline 3 & 50.40769 & 1 \\
\hline 4 & 51.42107 & 1 \\
\hline 5 & 52.42615 & 1 \\
\hline 6 & 53.41045 & 1 \\
\hline 7 & 54.46201 & 1 \\
\hline 8 & 55.52096 & 1 \\
\hline 9 & 57.42808 & 1 \\
\hline 10 & 59.49049 & 1 \\
\hline 11 & 61.51616 & 1 \\
\hline 12 & 62.45108 & 1 \\
\hline 13 & 306.55380 & 1 \\
\hline 14 & 316.59299 & 1 \\
\hline 15 & 324.53348 & 1 \\
\hline 16 & 347.57980 & 1 \\
\hline 17 & 350.52344 & 1 \\
\hline 18 & 395.46509 & 1 \\
\hline 19 & 397.53473 & 1 \\
\hline 20 & 400.56161 & 1 \\
\hline 21 & 403.41821-403.50235 & 2 \\
\hline 22 & 404.42032 & 1 \\
\hline 23 & 405.44067 & 1 \\
\hline 24 & 406.43344 & 1 \\
\hline 25 & 408.46484 & 1 \\
\hline 26 & 434.35504 & 1 \\
\hline 27 & 440.29681 & 1 \\
\hline 28 & 443.32071 & 1 \\
\hline 29 & 449.37424 & 1 \\
\hline 30 & 522.26080 & 1 \\
\hline 31 & 530.24188 & 1 \\
\hline 32 & 531.26245 & 1 \\
\hline 33 & 600.69812 & 1 \\
\hline 34 & 749.37793-749.59137 & 12 \\
\hline 35 & $750.37024-750.61023$ & 19 \\
\hline 36 & $751.36682-751.61029$ & 21 \\
\hline 37 & $752.37366-752.62543$ & 25 \\
\hline 38 & 753.37134-753.62561 & 43 \\
\hline 39 & $754.36322-754.63074$ & 38 \\
\hline 40 & $755.35388-755.62952$ & 38 \\
\hline
\end{tabular}

With this experimental setup, the star's projected rotation velocity of $46 \mathrm{~km} \mathrm{~s}^{-1}$ implies a resolution of $5.5 \mathrm{~km} \mathrm{~s}^{-1}$ and gives about 16 effective points in the line profile, sufficient to ensure that modes up to $|m|<15$ would be detected (see Sect. 4). The average timespan between subsequent exposures of this dataset corresponds to a "Nyquist" frequency above $50 \mathrm{~d}^{-1}$. The data reduction was done in the usual manner using standard IRAF ${ }^{1}$ procedures, except for the final continuum normalization which was done using our own software. The mean $\mathrm{S} / \mathrm{N}$ of the spectra is 250. Table 1 lists the log of our observations.

Since the observed intensity of LPV is about $2 \%$ of the continuum, its determination is an important step in data reduction. Spectra were first normalized to the continuum by using a cubic spline function. The average spectrum was then calculated and used to adjust the individual spectra by fitting a low order ( 2 or 3 ) polynomial to the continuum points in each spectrum. Note that, for rapidly rotating stars, this procedure leads to the

${ }^{1}$ IRAF is distributed by the National Optical Astronomy Observatories, which is operated by the Association of Universities for Research in Astronomy (AURA), Inc., under cooperative agreement with the National Science Foundation.

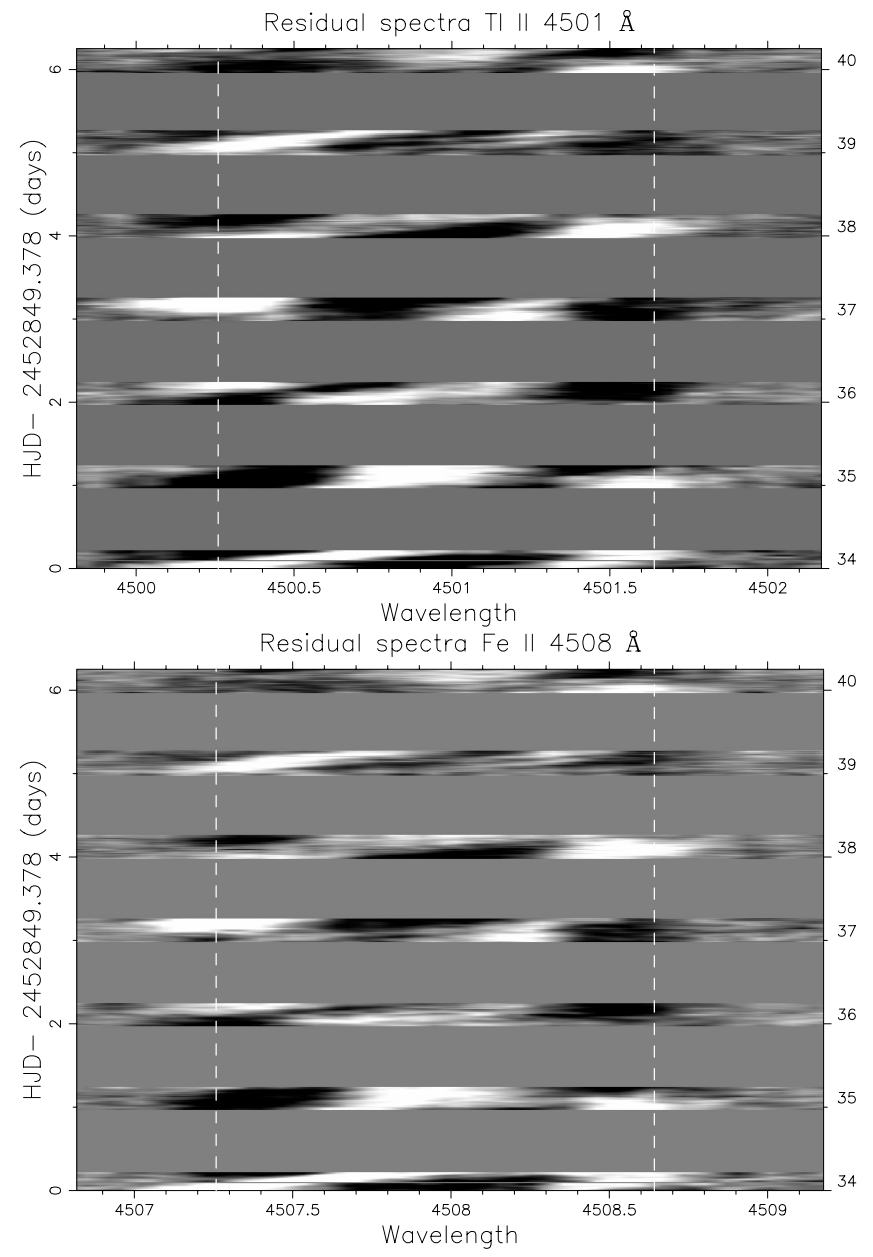

Fig. 1. Dynamic residual spectra (average spectrum subtracted) taken on 7 consecutive nights. Vertical lines indicate the wavelengths corresponding to the projected rotational velocity $\pm 46 \mathrm{~km} \mathrm{~s}^{-1}$. The upper panel shows the Ti II $4501 \AA$ line while the Fe II $4508 \AA$ line is shown in the lower panel.

determination of a quasi-continuum since rotationally broadened low intensity lines tend to depress the level of the observed continuum with respect to the true continuum. Furthermore, rotationally broadened synthetic or template spectra are generally more appropriate as reference continua. However, since we used only the residuals (average spectrum subtracted from an individual spectrum) the absolute value of the continuum is unimportant and the use of an average spectrum as a reference is a correct procedure.

When considering individual lines, we mainly concentrated on two unblended ones, Ti II and Fe II at respectively 4501.273 and $4508.288 \AA$. Figure 1 shows gray-scale representations of the time series of the residual spectra with respect to the average profile, obtained during the 7 consecutive nights for both lines. Note that the behavior of these two different lines shows excellent agreement.

\section{Time series analysis}

\subsection{Radial velocities}

Radial velocities are in the range $[-30 ;-20] \mathrm{km} \mathrm{s}^{-1}$, comparable to what is reported in Paper I. The radial velocity curves generally have quite a large dispersion that can prevent frequency analysis. Therefore, we computed the correlation profiles using 


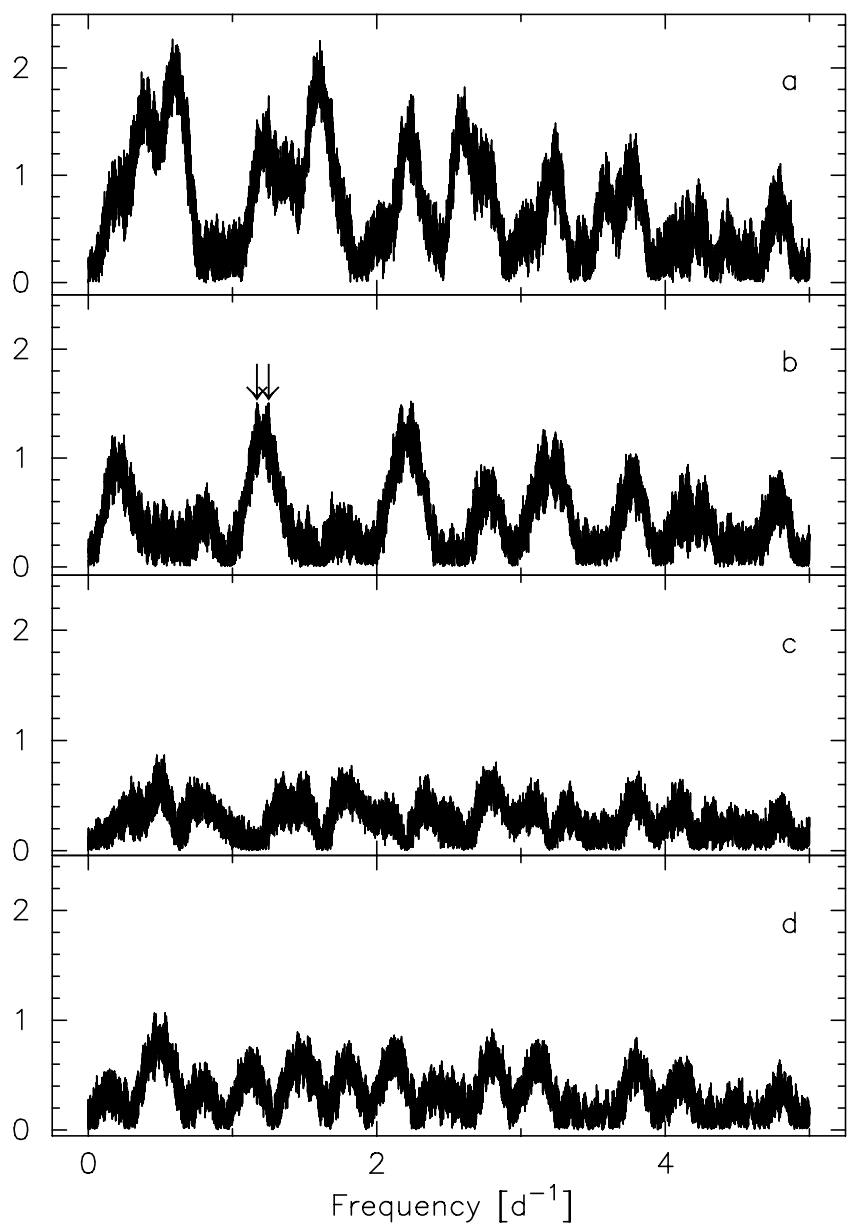

Fig. 2. Frequency analysis performed on the velocities related to the first moment computed from the correlation profile. a) Standard Fourier analysis performed on the whole data set; b) standard Fourier analysis performed on the same data set after prewhitening with the $1.61 \mathrm{~d}^{-1}$ frequency (see text). The two arrows represent the 1.25 and the $1.17 \mathrm{~d}^{-1}$ frequencies; c) same analysis performed on the residual data set after prewhitening simultaneously with the $1.61,1.25$ and $1.17 \mathrm{~d}^{-1}$; d) analysis after prewhitening simultaneously with the $1.61,1.25$ and $1.30 \mathrm{~d}^{-1}$ frequencies.

an artificial mask containing about 20 metallic lines. In the following, we make the reasonable assumption that all lines considered in the mask vary simultaneously in the same way, and provide a reliable mean profile allowing a better determination of the radial velocity curves and consequently of the frequency spectrum (Le Contel et al. 2001). This spectrum is computed from the first moment of the correlation profile.

We first performed a standard Fourier analysis on the whole data set. The corresponding results are represented in Fig. 2. The peak corresponding to the Hipparcos $1.25 \mathrm{~d}^{-1}$ frequency is not dominant in our data. Instead, two stronger peaks are present, at respectively 0.61 and $1.61 \mathrm{~d}^{-1}$, which are 1 -d aliases of each other. For reasons given below, we retained the $1.61 \mathrm{~d}^{-1}$ frequency, which has an amplitude $K_{1}=2.15 \mathrm{~km} \mathrm{~s}^{-1}$, and accounts for about $40 \%$ of the variance of the corresponding sine-fit. After prewhitening with $1.61 \mathrm{~d}^{-1}$, the $1.2506 \mathrm{~d}^{-1}$ frequency is easily detected, with a precision close to that of Hipparcos $\left(1.25051 \mathrm{~d}^{-1}\right.$, associated with an amplitude of $37 \mathrm{mmag}$ ). However, this precision is only indicative, since there is uncertainty about which peak to choose due to our data sampling. In addition, the peak presents a double structure,
Table 2. Results provided by the sine-fit with the 4 frequencies simultaneously applied to the correlation data set. Columns represent respectively the frequency and the $K$ amplitude $\left( \pm 0.18 \mathrm{~km} \mathrm{~s}^{-1}\right)$.

\begin{tabular}{cc}
\hline \hline Frequency $\left[\mathrm{d}^{-1}\right]$ & $K\left[\mathrm{~km} \mathrm{~s}^{-1}\right]$ \\
\hline 1.61 & 1.47 \\
1.25 & 3.31 \\
1.30 & 2.28 \\
0.96 & 0.58 \\
\hline
\end{tabular}

and a second frequency is present at $1.17 \mathrm{~d}^{-1}$. Like $1.61 \mathrm{~d}^{-1}$, this frequency is not mentioned in the photometric study by Henry et al. (2005), involving a 1-year observation data set. In addition, a sine-fit performed on the whole data set with $1.25,1.61$ and $1.17 \mathrm{~d}^{-1}$ accounts for about $70 \%$ of the variance, $1.17 \mathrm{~d}^{-1}$ accounting for it by less than $10 \%$. Imposing the photometric frequency $1.30 \mathrm{~d}^{-1}$ leads to a similar result. Therefore, we favor the presence of $1.61 \mathrm{~d}^{-1}$, and then the 3 other photometric frequencies $1.25,1.30$ and $0.97 \mathrm{~d}^{-1}$ (Henry et al. 2005) in our data set, the $1.17 \mathrm{~d}^{-1}$ frequency being due to our bad sampling. Quantities concerning these 4 frequencies are given in Table 2.

The total fraction of the variance is about $70 \%$, which means that additional frequencies are certainly present. The Fourier spectrum after prewhitening with the main considered frequencies does not show any region with significant power (see Fig. 2), so the additional frequencies may be of an amplitude level that is below the noise. Note also that Henry et al. (2005) pointed out the presence of a Blazhko effect in this star. Therefore, dispersion is present around the phase of maximum light, and may also be present in spectroscopic data.

It is also shown in Table 2 that the amplitude associated with the $1.61 \mathrm{~d}^{-1}$ frequency is much lower than the amplitudes related to 1.25 and $1.30 \mathrm{~d}^{-1}$. The fact that $1.61 \mathrm{~d}^{-1}$ is detected is due to the beating effect between 1.25 and $1.30 \mathrm{~d}^{-1}$, which canceled each other at the end of the 7 consecutive nights. Our data are spread over more than 2 years, but most measurements were obtained during the last 7 consecutive nights. Therefore, this epoch has a huge weight in the data analysis. The beat period between 1.25 and $1.30 \mathrm{~d}^{-1}$ is around $20 \mathrm{~d}$, and should modulate the signal present within these 7 consecutive nights (Fig. 3). Note that the peaks associated with 0.61 and $1.61 \mathrm{~d}^{-1}$ are very similar, and account respectively for $38 \%$ and $40 \%$ of the variance if a sine-fit is considered. Figure 4 represents the data corresponding to the 3 last nights phased with the 0.61 and $1.61 \mathrm{~d}^{-1}$ frequencies. It is clear that the frequency $1.61 \mathrm{~d}^{-1}$ is best suited to account for the data variations.

\subsection{Line profiles}

The high data quality allowed us to perform a frequency analysis and a preliminary mode identification directly on the line profile variations. However merging of different spectral lines can introduce erroneous results. In order to avoid this problem, we only use unblended Fe II and Ti II lines. Due to the very good temporal sampling, we only take into account the observations from the seven consecutive nights presented in Fig. 1. There is of course an ambiguity in considering an identification for 2 close modes having quite different amplitudes (Table 2). Despite the fact that no clear identification can be made to correspond to the $1.25 \mathrm{~d}^{-1}$ and to the $1.30 \mathrm{~d}^{-1}$ frequencies (these frequencies not being resolved within the 7 night range), we consider only the case corresponding to the so-called "intermediate" frequency at $1.27 \mathrm{~d}^{-1}$. The corresponding two-dimensional Fourier transform 


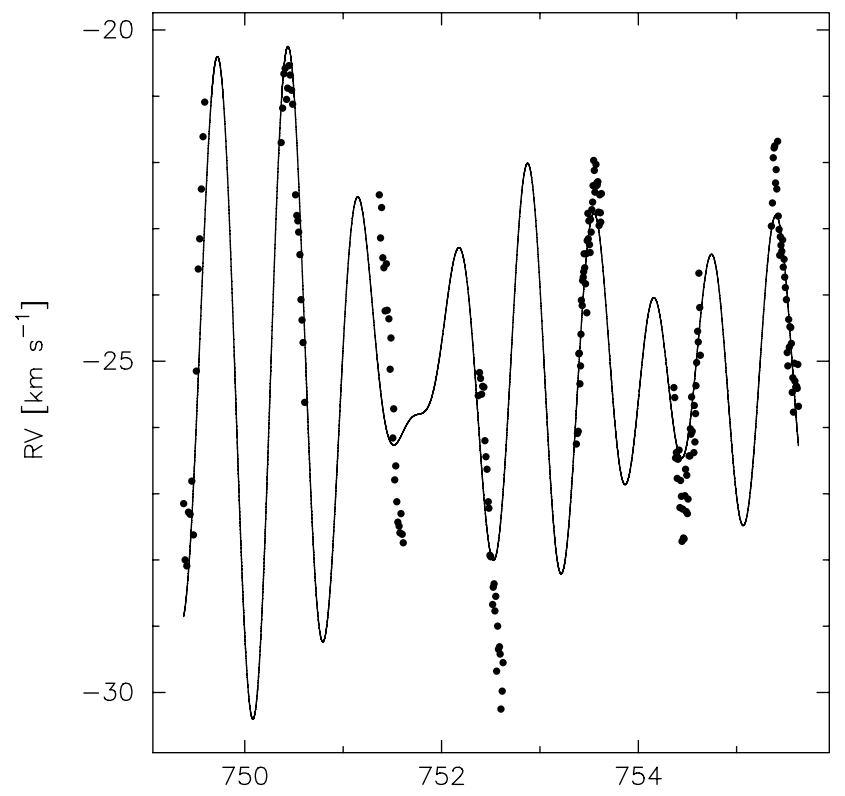

HJD

Fig. 3. Heliocentric radial velocities for the 7 consecutive nights. The curve represents the sine-fit using the 1.611 .25 and $1.30 \mathrm{~d}^{-1}$ frequencies.

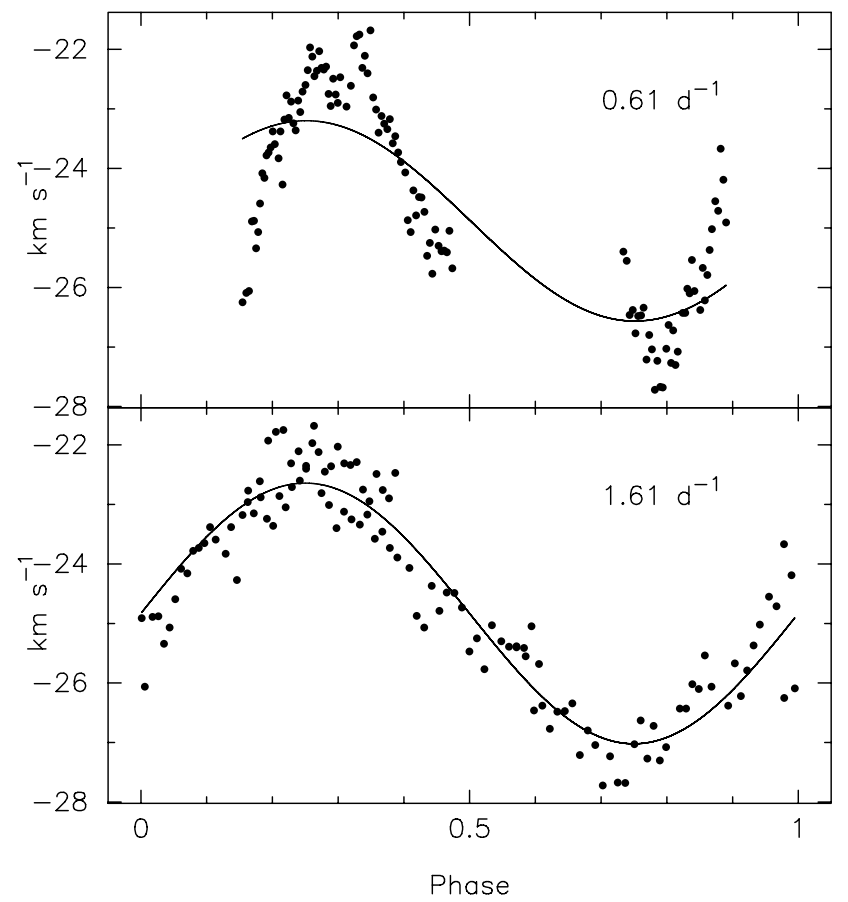

Fig. 4. Heliocentric radial velocity data phased with the 0.61 and $1.61 \mathrm{~d}^{-1}$ frequencies, together with the corresponding sine-fit.

is plotted in Fig. 5 in which one can see that there is considerable power near periods around $0.3,0.6,1.3,1.6,2.3,2.6 \mathrm{~d}^{-1} \ldots$, and normalized wavelength frequencies (see next section) in the $\ell=3-5$ range. For each wavelength frequency in that range we performed a time-series analysis in order to remove the temporal window function (which is due to incomplete temporal sampling of the variational signal). Using the window function which describes how the power from the true frequency is

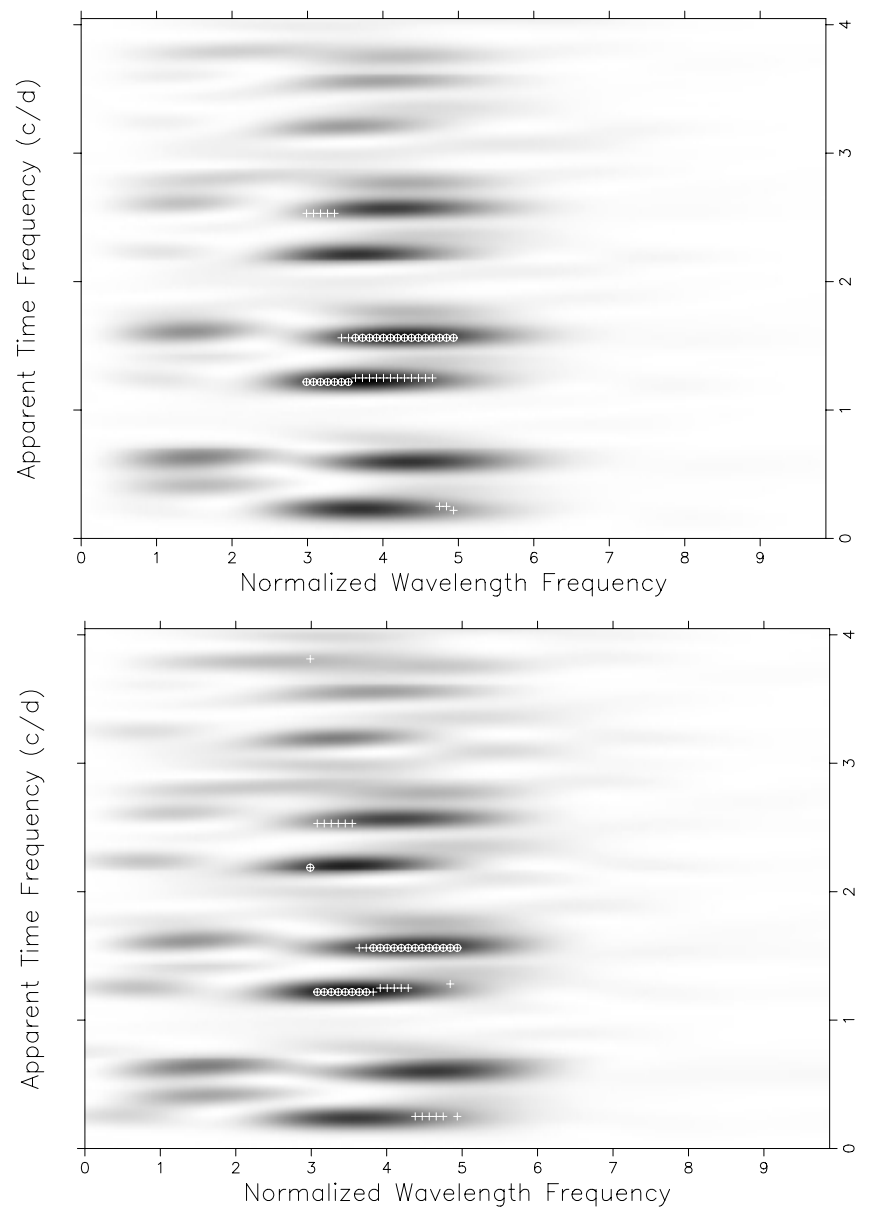

Fig. 5. Frequency analysis of the LPV in selected spectral lines using the CLEANEST algorithm searching for up to 2 independent frequencies in each normalized wavelength frequency bin. Two-dimensional Fourier diagrams ("dirty spectrum") are shown for Ti II $4501 \AA$ (top) and Fe II $4508 \AA$ (bottom) where the found values ("clean spectrum") are presented as crosses and dotted circles. The frequencies at 1.27 and $1.61 \mathrm{~d}^{-1}$ are present for both lines.

redistributed among neighboring frequencies, we removed the alias patterns with the CLEANEST algorithm (Foster 1995). Up to 2 independent frequencies were searched for in the time series in each normalized wavelength frequency bin that contains the true frequencies as well as aliases ("dirty spectrum"). The frequencies found by the algorithm ("clean spectrum") are presented as crosses and dotted circles in Fig. 5. It is clear that for both lines the algorithm found two dominant frequencies: 1.61 and the intermediate $1.27 \mathrm{~d}^{-1}$, which correspond to the frequencies detected in radial velocity variations.

\section{Mode identification}

\subsection{Moment method}

In this method, the first 3 velocity moments of the line profiles are calculated, and the most probable solution is found when the corresponding discriminant attains a minimum (Balona 1987; Aerts 1996). The main advantage of this method with respect to the FDI method (see below) is that in principle it can provide the complete set of NRP parameters, such as stellar inclination and the pulsational velocity (and/or temperature) amplitudes and phases. The FDI method does not give the complete set of parameters. Consequently, the observed line profiles cannot be 
modeled. This in turn means that the observed light amplitude cannot be used as a constraint on mode identification (Balona 1998). In order to obtain the complete set of NRP parameters, we applied the method of moments to our data.

The discriminant values are very close to each other, even for the dominant frequency $1.61 \mathrm{~d}^{-1}$, and only the radial mode can actually be excluded from such an analysis. We did not attempt to apply the moment method to the intermediate frequency since the moments would be polluted by the mode corresponding to $1.61 \mathrm{~d}^{-1}$.

The most probable explanation for the failure of the method is that the high degree modes dominate in our spectroscopy data. Carrying out a theoretical study of the accuracy, De Pauw et al. (1993) found that the method of moments was able to identify low degree $(\ell<4)$ modes but not the $\ell=4(|m|=1,2,3,4)$ ones. In almost all cases they had a list of several modes, with the same minima for the discriminant. As a result of this, they found it was impossible to identify the correct mode. In this situation (as well as for higher values of $\ell$, since they expect the results to be similar to those of $\ell=4$ ) the only possible conclusion is that the mode has a degree larger than $\ell=3$. Generally speaking, the moment method is preferred for low values of $\ell$ and Doppler imaging for high values of $\ell$ (Balona 1998).

\subsection{Doppler mapping of non-radial pulsations}

The non-radial pulsator model describes the surface intensity distribution and velocity field perturbations in terms of the associated Legendre functions $P_{\ell}^{m}\left(b, \phi_{\mathrm{R}}\right)$ and time $t$ as:

$A_{\mathrm{I}} P_{\ell}^{m}(\sin b) \mathrm{e}^{\mathrm{i}\left(m \phi_{\mathrm{R}}+\omega t\right)}$,

where $A_{\mathrm{I}}$ is the oscillation amplitude, $\omega=2 \pi f$ is the angular time frequency in the observer's frame, the quantum numbers $(\ell, m)$ denote the pulsation degree and azimuthal order, respectively, $b$ is the latitude and $\phi_{\mathrm{R}}$ is the azimuth related to the star's rotational frequency $\Omega_{R}$. The rotational phase should be related to the NRP wave frequency $\Omega_{\mathrm{W}}$ defined as:

$m \Omega_{\mathrm{W}} t=m \phi=m \phi_{\mathrm{R}}+\omega t$,

where $\phi$ is the corresponding azimuth defined by the same relation.

In rapid rotators, there is a one-to-one mapping between the points in the broadened line profile and the position of perturbations on the stellar surface (Vogt \& Penrod 1983). The technique of "Doppler mapping" led to various methods to identify pulsation parameters (degree $\ell$ and order $m$ ) from high-resolution, high signal-to-noise spectroscopic observations. However, no attempts have been made to determine the latitude $b$ where the pulsation amplitude is maximum at the stellar surface. Since for the sectoral modes $(|m|=\ell)$ most of the variability is located at the equator of the star, and for tesseral modes $(|m| \neq \ell)$ the main source of variability lies off the equator, the determination of $b$ is closely related to the modal analysis.

The photospheric absorption line in which rotation is the dominant broadening mechanism displays time-variable perturbations ("bumps" or "dips") which are carried (as the star rotates) across the stellar disk. Consequently, the bumps change their Doppler shifts in accordance with their projected distances from the stellar rotation axis which depends on azimuth $\phi$ :

$\Delta \lambda=\frac{\lambda}{\mathrm{c}} V_{\mathrm{e}} \sin i \cos b \sin \phi$,

where the projected rotational velocity $V_{\mathrm{e}} \sin i$ is known, for example from Fourier analysis of the average profile. Relating the azimuth to time $t$ through $\phi=\frac{\omega}{m} t$, the "bump" paths, corresponding to the observed frequency $f$, can be traced in the dynamic (wavelength/time) spectrum. In addition, the NRP phase $\Phi$ can be measured by fitting the sinusoids in each wavelength bin of the dynamic spectrum. The phase diagrams (wavelength $/ \Phi$ ) can be used to determine $b$, relating $\phi$ (in Eq. (2)) to $\Phi$ through: $\phi=\frac{\Phi}{m} 2 \pi$. From the above equations it is obvious that the "bump" paths, as well as NRP phases, trace out a sinusoidal variation with an amplitude equal to $V_{\mathrm{e}} \sin i \cos b$, from which $b$ can be deduced if $V_{\mathrm{e}} \sin i$ is known. This value of $b$ depends also on $m$, and should be consistent with the value of $\ell$ that can be determined using other methods (e.g. Fourier-Doppler Imaging).

\subsection{Fourier-Doppler imaging}

This approach leads to a two-dimensional Fourier analysis in both time and space and its main advantage is that it decomposes complex line profile variations represented as a sum of terms in Eq. (1) into single components. The wavelength is mapped on stellar longitude $\phi$ using:

$\phi_{k}=\sin ^{-1}\left(\frac{c}{\lambda} \frac{\Delta \lambda_{k}}{V_{\mathrm{e}} \sin i} \frac{1}{\cos b}\right)$

where $\Delta \lambda_{k}$ is the Doppler shift with respect to the line center, corresponding to the wavelength bin $k$.

Assuming that the oscillations are confined to the equatorial region $(\cos b \approx 1)$, which is particularly true for sectoral $\ell=|m|$ oscillations, the normalized wavelength frequency can be associated with the azimuthal order of the pulsation modes (Kennelly et al. 1992). In the general case, when tesseral modes are present, the normalized wavelength frequency represents the non-radial degree $\ell$ more closely than the azimuthal order $m$ (Kennelly et al. 1996). Although the method is best suited for very rapid rotators, in their application to $\theta^{2}$ Tauri, Kennelly et al. (1996) showed that the method can be successfully applied to a star with moderate projected rotational velocity $\left(V_{\mathrm{e}} \sin i=70 \mathrm{~km} \mathrm{~s}^{-1}\right)$ even for high degree $\ell \leq 10$ modes. Our numerical simulations for a star rotating with $V_{\mathrm{e}} \sin i=45 \mathrm{~km} \mathrm{~s}^{-1}$ and assuming a typical intrinsic line width of $\sim 5 \mathrm{~km} \mathrm{~s}^{-1}$ and our spectral resolution ( $2.7 \mathrm{~km} \mathrm{~s}^{-1}$ per pixel) showed that the considered moderate degree modes $\ell=3$ and $\ell=7$ could be successfully identified, consequently we conclude that the same applies to any $3 \leq \ell \leq 7$ mode in HD 195068.

\subsection{Application to the frequency $1.61 \mathrm{~d}^{-1}$}

Although the star's pulsation is multi-periodic and complex, the line-profile variabilities caused by the individual pulsation modes were separated in frequency as a result of the Fourier analysis which allows the characteristics of this mode to be studied separately. However, even though the modes are separated in frequency, the possible mode interaction cannot be completely ignored. For this reason we folded the data with the considered frequency, a technique that in principle cancels out other frequencies except harmonics. Moreover for the modal analysis of the $1.61 \mathrm{~d}^{-1}$ frequency we used the end of the 7 days run (nights $37-40$ from Table 1), while for $1.27 \mathrm{~d}^{-1}$ we used the beginning (nights 34-37), the time intervals when the corresponding frequencies were dominant (see Fig. 10).

The dynamic spectrum of residuals (average spectrum subtracted) folded with $1.61 \mathrm{~d}^{-1}$ is presented in Fig. 6 (top-left) where the "bump" paths correspond to a $|m|=4$ mode confined 

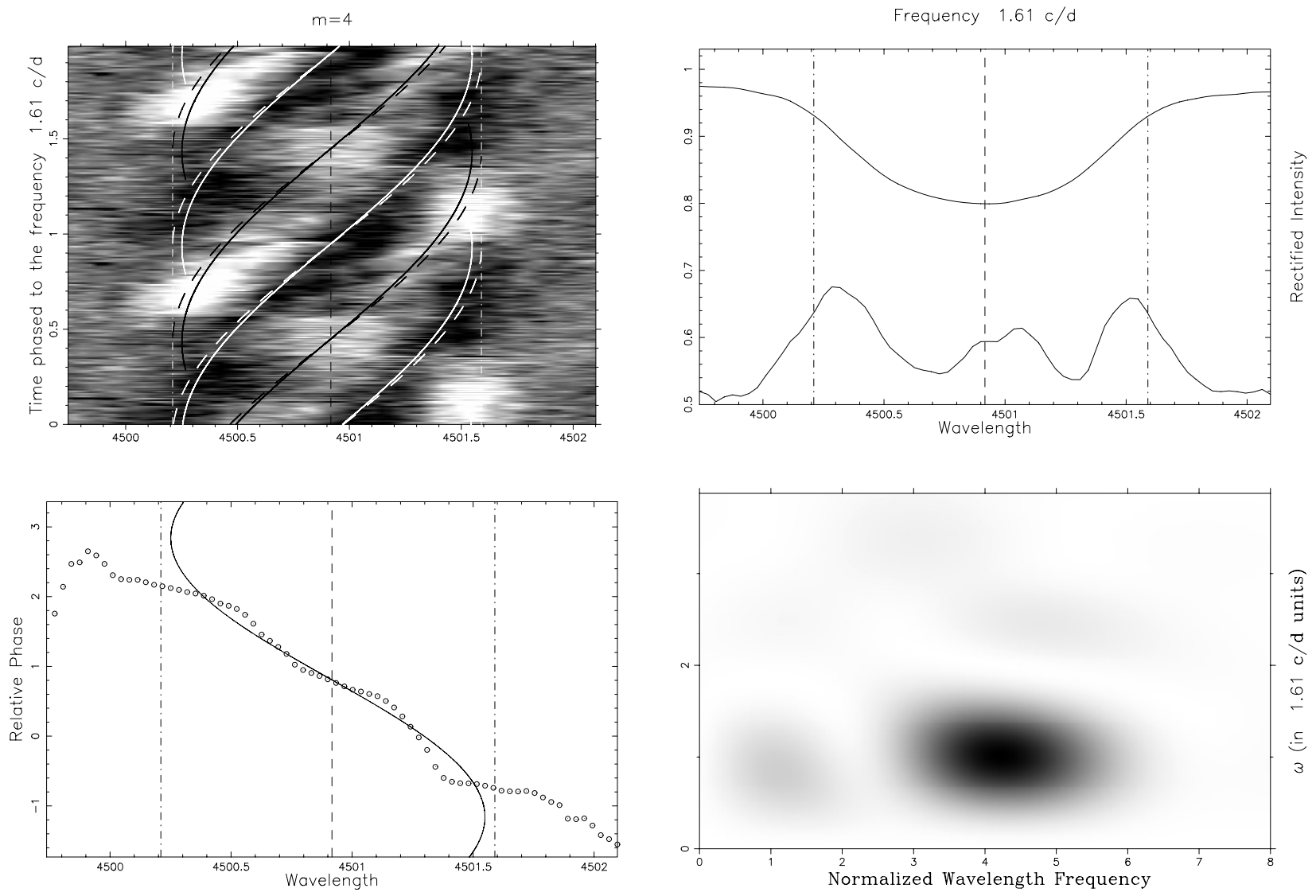

Fig. 6. Top-left: dynamic spectrum of residuals folded with the frequency $1.61 \mathrm{~d}^{-1} \cdot|m|=4$ mode at $b=0^{\circ}$ (dashed) and $b=20^{\circ}$ (full). Bottom-left: NRP phases and sinusoidal fit. Bottom-right: FDI. Top-right: NRP amplitudes and average profile.

to the equator (dashed lines) or to the latitude $b=20^{\circ}$ (full lines). In the bottom-left panel the measured NRP phases (in $2 \pi$ radians) are plotted as circles, while the full line represents the sinusoidal fit which corresponds to $b=20^{\circ}$. Note that the Doppler imaging technique does not assume a priori that the pulsation is most dominant at the equator and stellar latitude was fixed from Eq. (2) using $V_{\mathrm{e}} \sin i=46 \mathrm{~km} \mathrm{~s}^{-1}$ (Paper I). Because of the fact that the measured value of $V_{\mathrm{e}} \sin i$ includes the star's inclination angle the deduced latitude does not depend on this parameter. The Fourier-Doppler diagram is plotted at the bottom-right of the figure, while the measured NRP amplitudes across the line profile are presented in the top-right. The analysis of FDI diagrams implies $\ell=4.5 \pm 1$ and we explored the parameter space only for $|m|=4 \pm 1$ since it produced better fits than $|m|<3$. Figure 7 shows the "bump" (top) and phase (bottom) paths corresponding to the mode $|m|=3$ (left) and $|m|=5$ (right). Full lines correspond to $b=35^{\circ}(|m|=3)$ and $b=0^{\circ}(|m|=5)$, and dashed lines correspond to the equator. Note that for the $|m|=5$ mode the fitted amplitude in the phase diagram is larger than the $V_{\mathrm{e}} \sin i=46 \mathrm{~km} \mathrm{~s}^{-1}$ limit which is shown as vertical dashed lines. This mode should be excluded since it implies $\cos b>1$. Consequently, for the best $|m|=4$ value the mode $\ell=5$ is more consistent with the deduced latitude $b=20^{\circ}$ than $\ell=4$.

Note that we only present the results for the Ti II $4501 \AA$ line, since results for the Fe II $4508 \AA$ line are essentially the same. For the same reason we do not present results corresponding to different published values of $V_{\mathrm{e}} \sin i$ (e.g. $43 \mathrm{~km} \mathrm{~s}^{-1}$ (Abt $\&$ Morrell 1995) and $52 \mathrm{~km} \mathrm{~s}^{-1}$ (Royer et al. 2002)) since they imply $\ell \sim 4.3$ and $\ell \sim 4.9$ respectively which is smaller than the formal error $( \pm 1)$ associated with the $\ell$ values.

\subsection{Application to the $1.27 d^{-1}$ intermediate frequency}

The NRP pattern for the intermediate frequency can be seen clearly in the folded spectra (Fig. 8 top-left).

The "bump" paths correspond to a $|m|=3$ mode confined to the equator (dashed lines) or to the latitude $b=35^{\circ}$ (full lines). The vertical dashed lines are drawn at the wavelengths corresponding to $\pm V_{\mathrm{e}} \sin i$. At the bottom-left the measured NRP phases across the line profile are plotted as circles, while the full line represents the sinusoidal fit which corresponds to $b=35^{\circ}$. The Fourier-Doppler diagram is plotted at the bottom-right of the figure, while the measured NRP amplitudes are presented at the top-right. Figure 9 shows the "bump" (top) and phase (bottom) paths corresponding to the mode $|m|=2$ (left) and $|m|=4$ (right). The full lines correspond to $b=40^{\circ}(|m|=2)$ and $b=10^{\circ}(|m|=4)$, and dashed lines correspond to the equator. Since the normalized wavelength frequency in FDI diagrams is an indicator of the degree $\ell$, our analysis implies $\ell=4 \pm 1$ and for this reason the parameter space has only been explored for $|m|=3 \pm 1$ which gives much better fits than $|m|<2$.

Since this identification corresponds to an instantaneous superposition of 1.25 and $1.30 \mathrm{~d}^{-1}$ modes it is not excluded that the $1.25 \mathrm{~d}^{-1}$ (and/or $1.30 \mathrm{~d}^{-1}$ ) mode corresponds to a lower degree than the effectively measured value of $\ell=4 \pm 1$. This would reconcile both the spectroscopic and photometric points of view, since this latter technique favors the detection of low degree modes, and Hipparcos was able to detect the $1.25 \mathrm{~d}^{-1}$ frequency. 

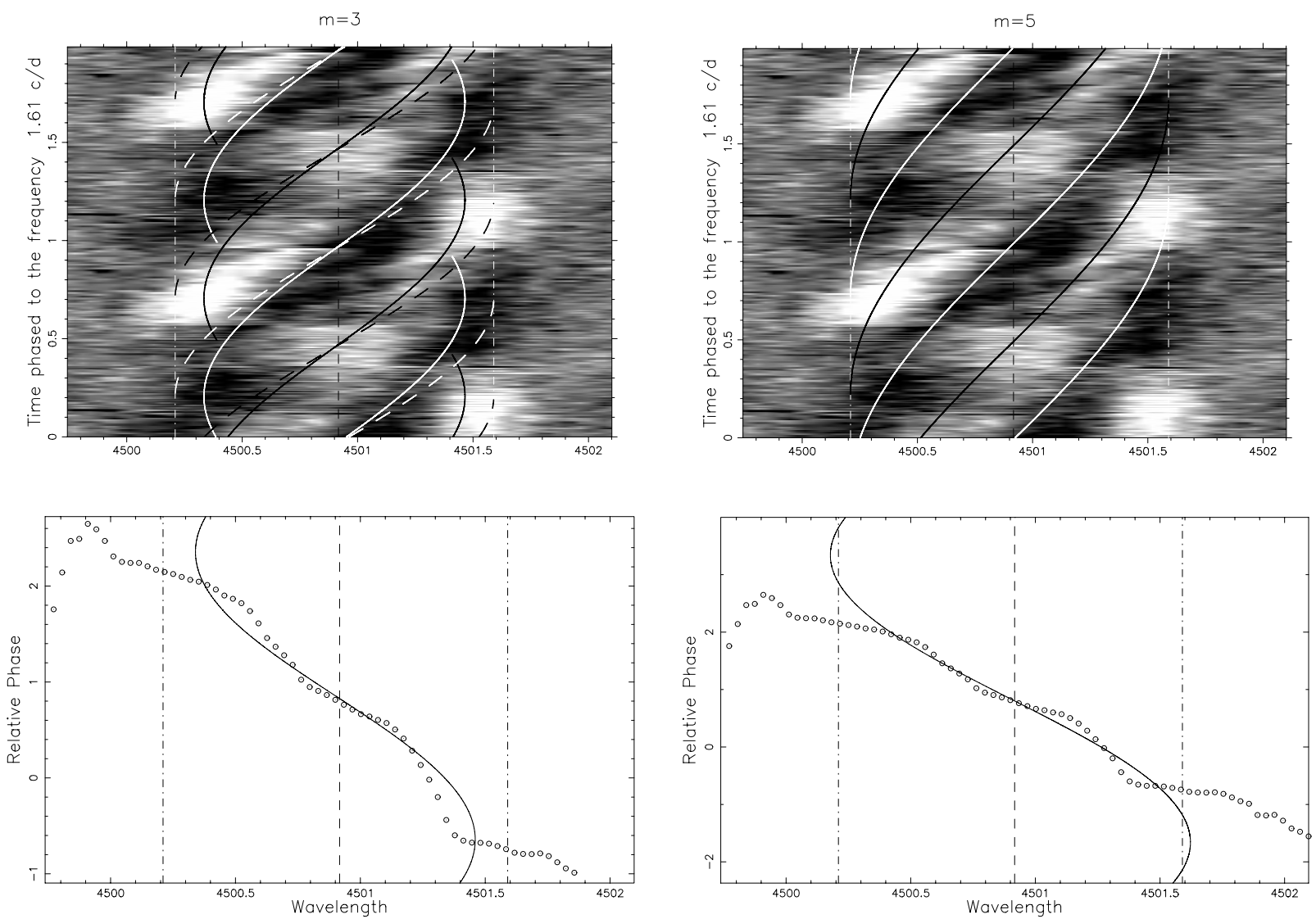

Fig. 7. The "bump" (top) and phase (bottom) paths corresponding to modes $|m|=3$ (left) and $|m|=5$ (right). The full lines correspond to $b=35^{\circ}$ $(|m|=3)$ and $b=0^{\circ}(|m|=5)$.

$m=3$
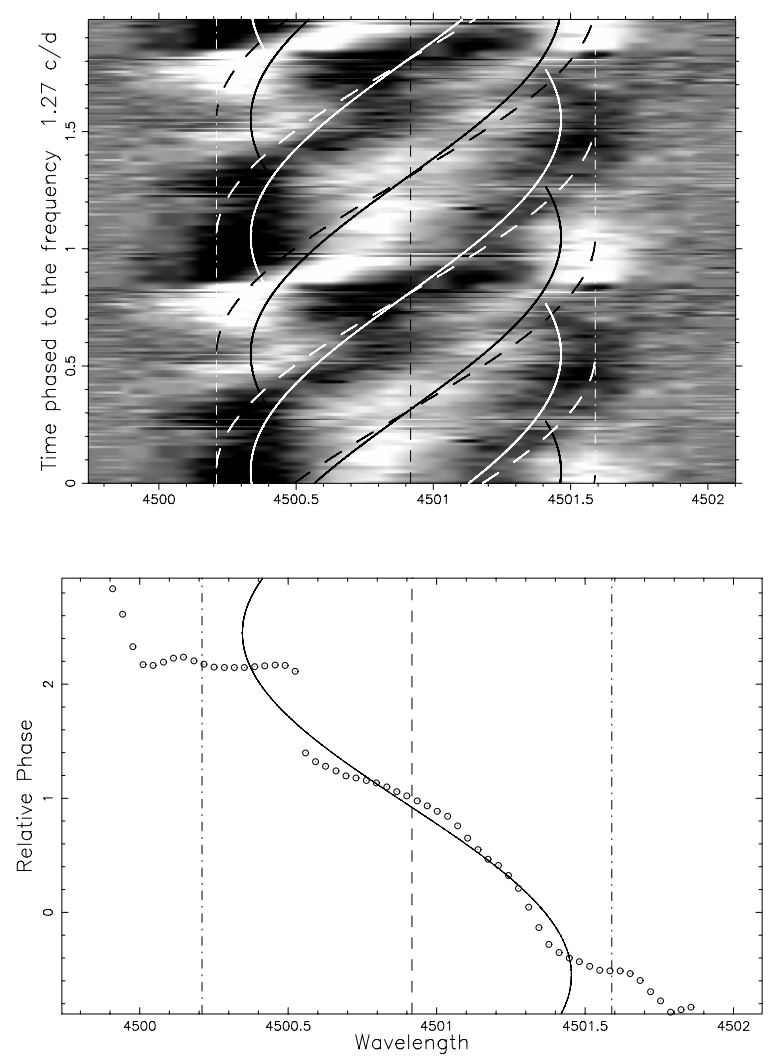

Frequency $1.27 \mathrm{c} / \mathrm{d}$
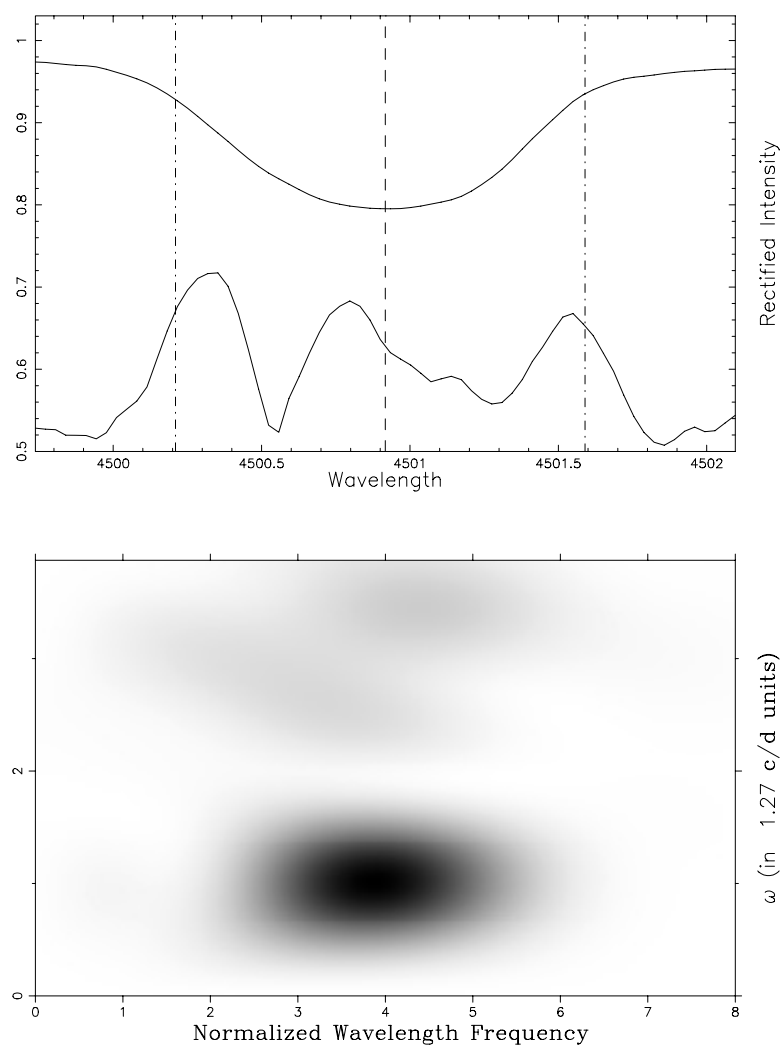

Fig. 8. Top-left: dynamic spectrum of residuals folded with the intermediate frequency $1.27 \mathrm{~d}^{-1}$, with the superposition of mode $|m|=3$ at $b=0^{\circ}$ (dashed) and $b=35^{\circ}$ (full). Bottom-left: NRP phases and sinusoidal fit. Bottom-right: FDI. Top-right: NRP amplitudes and average profile. 

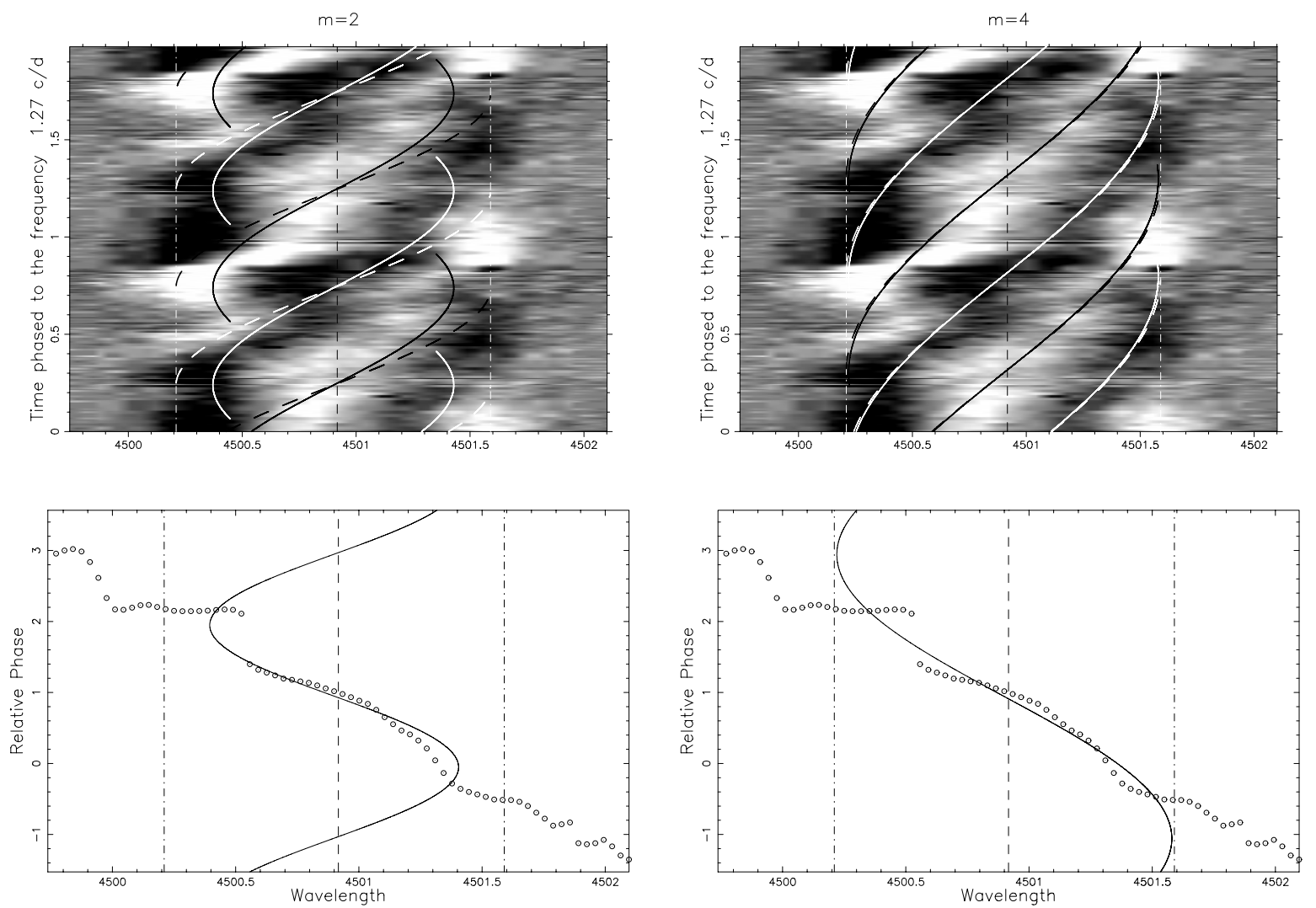

Fig. 9. The "bump" (top) and phase (bottom) paths corresponding to modes $|m|=2$ (left) and $|m|=4$ (right). The full lines correspond to $b=40^{\circ}$ $(|m|=2)$ and $b=10^{\circ}(|m|=4)$.

\section{Discussion}

\subsection{Modal nature of non-radial pulsations}

From Figs. 6 and 8 (top-right), we conclude that for both frequencies the variations appear to have a maximum strength at wavelengths symmetrical with respect to the line center, indicating a large $k_{n}$ (ratio of the horizontal to vertical velocity amplitude) value $\left(k_{n} \gg 1\right)$ since the maxima are very near the line wings.

This is a clear indication of low-frequency gravity modes because $k_{n}$ is related to the intrinsic pulsation frequency $\omega^{0}$ such that it is larger for short frequency pulsations (Osaki 1971):

$k_{n}=\frac{G M / R^{3}}{\left(\omega^{0}\right)^{2}}$

where $G$ is the gravitational constant and $M$ and $R$ are the stellar mass and radius. Such a distribution of pulsation amplitude is characteristic of high radial order $g$-modes while the maximum of the amplitude around line center is not, unless the associated temperature variations are large.

\subsection{Wave propagation and the rotation period}

It is obvious that the waves are prograde in the observer's frame because for such pulsations we expect that the variations of the non-radial mode will give rise to bumps and troughs that move from blue to red through the line profile. Equivalently, the phase diagrams of prograde modes have a negative slope from blue to red as can be seen in our phase diagrams.

However, the question of whether the waves are prograde in the stellar frame or not is closely related to the determination of the rotational frequency of the star. Accurate determination of the rotational frequency is not a straightforward task if the variability is associated with the waves moving in the co-rotating frame.

However, from the measured projected rotation velocity it is possible to relate the stellar rotational frequency to the stellar inclination $i$ and to the equatorial radius $R_{\mathrm{e}}$ by:

$\Omega_{\mathrm{rot}}=0.0198\left(V_{\mathrm{e}} \sin i\right) /\left(R_{\mathrm{e}} \sin i\right)$

where the numerical constants apply to the radius expressed in solar units and the period in days. With the observed $V_{\mathrm{e}} \sin i=$ $46 \mathrm{~km} \mathrm{~s}^{-1}$ and with $R=1.6 R_{\odot} \quad$ (Fekel et al. 2003), the obvious requirement $\sin i \leq 1$ implies $\Omega_{\mathrm{rot}} \geq 0.57 \mathrm{~d}^{-1}$.

Adopting $|m|=5,4,3$ for $1.61 \mathrm{~d}^{-1}$ we obtain for the frequency of each NRP wave $\Omega_{\mathrm{W}}=0.32,0.40,0.54 \mathrm{~d}^{-1}$ respectively, while adopting $|m|=4,3,2$ for $1.27 \mathrm{~d}^{-1}$ gives $\Omega_{\mathrm{W}}=0.32,0.42,0.64 \mathrm{~d}^{-1}$. Both modes are therefore retrograde in the stellar frame except if $|m|=2$ for $1.27 \mathrm{~d}^{-1}$. This combination can imply a corotating structure or a prograde wave in the stellar frame if $i>64^{\circ}$. However note that the phase diagram fit (Fig. 9 bottom-left) assuming $|m|=2$ for $1.27 \mathrm{~d}^{-1}$ is not satisfactory. Consequently the prograde mode is unlikely to be present in the star. Note also that the $|m|=5$ and $|m|=4$ solutions for $1.61 \mathrm{~d}^{-1}$ and $1.27 \mathrm{~d}^{-1}$ respectively imply the common frequency $\approx 0.32 \mathrm{~d}^{-1}$ which corresponds to the "super-period" of $\approx 3$ days as well as to the observed splitting $1.61-1.27=0.34 \mathrm{~d}^{-1}$. The existence of "super-periods" has already been reported for other types of non-radially pulsating stars (see e.g. Harmanec 1999) and, if this is the case for HD 195068, this definitively means that the observed modes are retrograde in the stellar frame.

\subsection{Apparent time variability of detected modes}

It has been noted in photometry that amplitude could change with time (see e.g. Poretti et al. 2002). In order to test their 

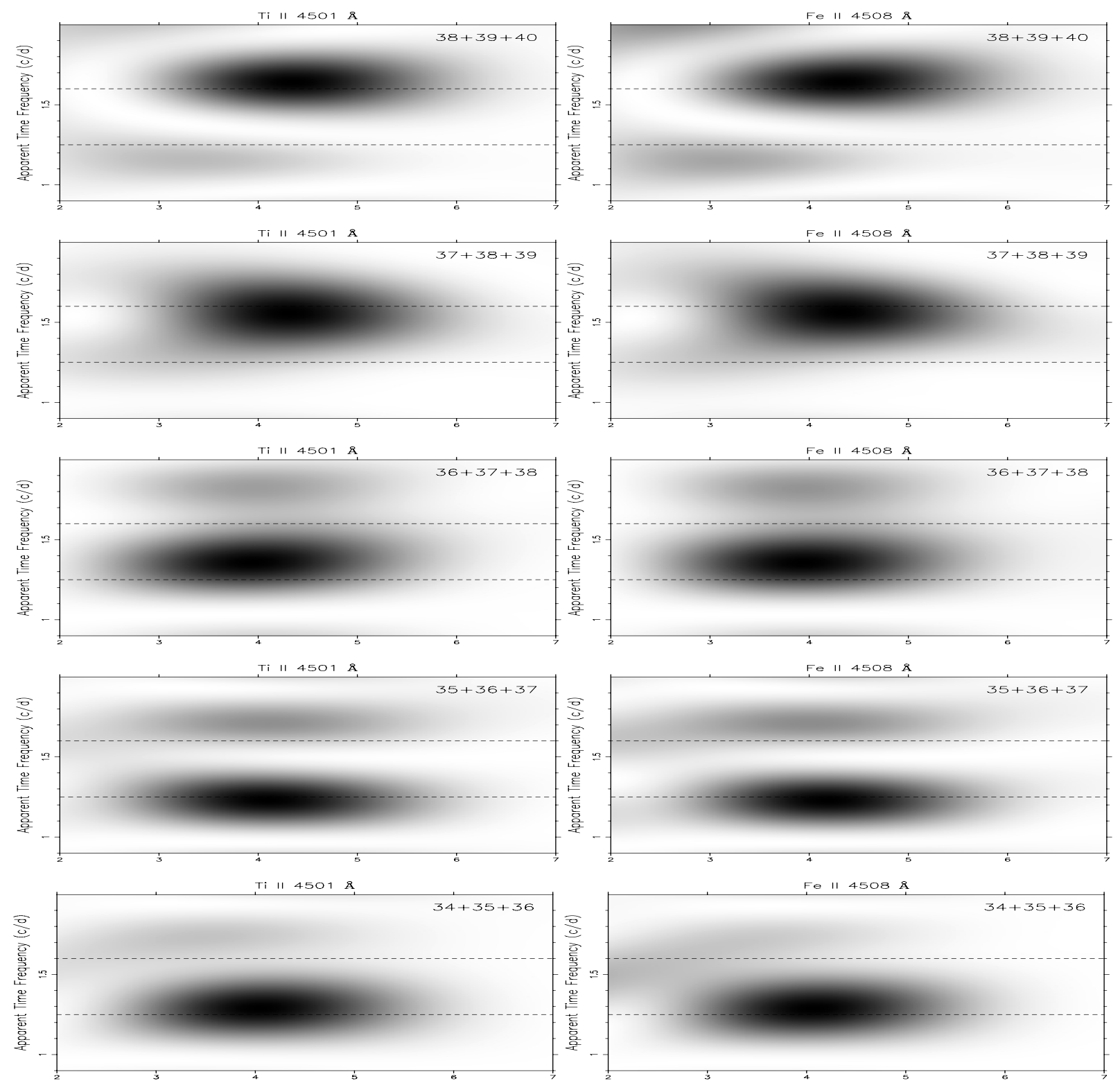

Fig. 10. Two-dimensional Fourier analysis of Ti II (left) and Fe II (right) using 3 consecutive nights listed in Table 1, as indicated at the top right of each figure. With respect to Fig. 5 the FDIs are zoomed to $0.9-2.0 \mathrm{~d}^{-1}$ and $\ell=2-7$ range. Horizontal dashed lines represent the $1.27 \mathrm{~d}^{-1}($ bottom) and $1.61 \mathrm{~d}^{-1}($ top $)$ frequencies. Note the switch of maximum power from $1.27 \mathrm{~d}^{-1}, \ell=4$ at the beginning of time series to $1.61 \mathrm{~d}^{-1}, \ell=4.5$ at the end.

stability, we studied the strength of both detected frequencies as a function of time. The typical duration of observations was 0.3 days per night and with a corresponding time resolution of $\approx 3 \mathrm{~d}^{-1}$ it was not possible to deduce the amplitude of two closely spaced $\left(\approx 0.3 \mathrm{~d}^{-1}\right)$ frequencies in a night-by-night sequence. However, using 3 consecutive nights, the frequencies are resolved, and moving a 3-night window in one night step through our time series, we performed the variability analysis for both detected frequencies. From the results presented in Fig. 10 it is obvious that maximum power switched from $1.27 \mathrm{~d}^{-1}, \ell=4$ at the beginning of spectroscopic observations to $1.61 \mathrm{~d}^{-1}, \ell=4-5$ at the end.

\subsection{Comparison with previous mode identifications in $\gamma$ Doradus stars}

Until now only a few attempts have been made to identify the pulsation modes in $\gamma$ Doradus stars. From line profile variations in $\gamma$ Doradus itself and using the moments method to identify the pulsational modes of the three periodic components, Balona et al. (1996) deduced the $(\ell, m)$ values of $(3,3)$ for $1.32098 \mathrm{~d}^{-1}$, $(1,1)$ for $1.36354 \mathrm{~d}^{-1}$ and $(1,1)$ for $1.47447 \mathrm{~d}^{-1}$ frequencies. For two frequencies $\left(0.346\right.$ and $\left.0.795 \mathrm{~d}^{-1}\right)$ detected on 9 Aurigae by Krisciunas et al. (1995), Aerts \& Krisciunas (1996) found with the moments method that they are a manifestation of the $\ell=3$, $|m|=1$ spheroidal mode and its toroidal corrections due to the rotation of the star. Investigating two frequencies $\left(1.32 \mathrm{~d}^{-1}\right.$ and $1.40 \mathrm{~d}^{-1}$ ) deduced from multisite photometry of HD 108100 , Breger et al. (1997) concluded that amplitude ratios and phase differences between the light curves at different wavelengths are consistent with $g$-mode pulsation of $\ell=1$, rather than $\ell=2$. For two of four frequencies observed in HD 164615, Zerbi et al. (1997) suggested an $\ell=3$ mode $\left(1.2328\right.$ and $\left.0.1301 \mathrm{~d}^{-1}\right)$, while the other two frequencies were associated with $\ell=2$ $\left(1.0899 \mathrm{~d}^{-1}\right)$ and $\ell=1\left(2.3501 \mathrm{~d}^{-1}\right)$ modes. Considering a single periodic variation $\left(1.2321 \mathrm{~d}^{-1}\right)$ in the star, Hatzes (1998) found that the $m=2$ mode seems to be consistent with the amplitude of the radial velocity variations. A preliminary mode identification 
based on the observed color curves of HD 218396 from a multisite campaign (Zerbi et al. 1999) indicated $\ell=2\left(1.9791 \mathrm{~d}^{-1}\right)$, $\ell=1\left(1.7268 \mathrm{~d}^{-1}\right)$ and either a $\ell=1$ or $\ell=2$ for $1.6498 \mathrm{~d}^{-1}$.

From simultaneous photometry and spectroscopy of the $\gamma$ Doradus variable HD 207223, Kaye et al. (1999b) have shown that the observed periodic (2.6 days) variations are the result of high-order $(n)$, low-degree $(\ell)$, non-radial $g$-mode pulsation. This star is an example that multiperiodicity is not observed in all $\gamma$ Doradus stars, and some of them display only a single photometric period (i.e. low-degree mode). Aerts \& Kaye (2001) confirmed that HD 207223 is also monoperiodic from a spectroscopic point of view. For the period 2.67 days they found an $\ell=m=+2$ solution.

Recently, Handler et al. (2002) determined the $\ell=|m|=1$ value for two observed $\gamma$ Doradus type non-radial pulsations (with frequencies 0.85 and $1.06 \mathrm{~d}^{-1}$ ) in the A-type primary of the eccentric binary HD 209295. Applying the method of Dupret et al. (2003) to the light curves of HD 12901 and HD 48501, Aerts et al. (2004) found that only an $\ell=1$ mode was able to explain the observed ratios for the three frequencies for the two stars for all wavelengths. Applying the FDI technique to the candidate $\gamma$ Doradus star HD 49434, Bruntt et al. (2002) detected the presence of one or several high-degree $(\ell \approx 6)$ as well as an $\ell \approx 2$ mode, but not present simultaneously. With the same technique Mathias et al. (2004b) determined an $\ell=3-4$ for a $1.94 \mathrm{~d}^{-1}$ frequency in HD 218396. As well as in two latter applications, our study of non-radial pulsations in HD 195068 reveals the relatively high degree modes $\ell \approx 4$. The previous photometrically-determined low degree $(\ell=1-3)$ modes in other $\gamma$ Doradus stars could be then just a consequence of the observational selection since the spectroscopy is much more sensitive to the intermediate and high degree non-radial pulsation modes.

\section{Conclusions}

We have presented time series of high-resolution spectra, showing line-profile variations of the Ti II $4501 \AA$ and Fe II $4508 \AA$ lines, in the $\gamma$ Doradus star HD 195068. Radial velocity analysis shows that 3 frequencies at least are present, namely 1.61, 1.25 and $1.30 \mathrm{~d}^{-1}$, the two last already having been detected photometrically. However, we were unable to detect the other frequency already observed in precise photometric observations, which has a considerably lower amplitude (a factor of 3 ) compared to the Hipparcos one (Henry et al. 2005). The presence of the $1.25 \mathrm{~d}^{-1}$ frequency was seen over more than 10 years, indicating a lower limit of the related mode lifetime. Because different filters are used, nothing can be concluded about the stability of the amplitude. The $2 K / \Delta m$ value associated with the Hipparcos frequency $1.25 \mathrm{~d}^{-1}$ is about $60 \mathrm{~km} \mathrm{~s}^{-1} \mathrm{mag}^{-1}$, a value in the range measured in Paper I.

The profile variations can be explained by non-radial pulsation modes, which definitively classify the star and confirm it to be a member of the $\gamma$ Doradus class. We analyzed the spectral time series by performing Fourier transforms and using the CLEANEST algorithm. This enabled us to identify 2 periods in the intensity variations across the line profiles. The dominant mode, corresponding to the $1.61 \mathrm{~d}^{-1}$ frequency, is identified as $\ell=5 \pm 1,|m|=4 \pm 1$. The mode corresponding to the intermediate $1.27 \mathrm{~d}^{-1}$ frequency can be modeled by the non-radial mode $\ell=4 \pm 1,|m|=3 \pm 1$.

Evidence that the star is not pulsating in the radial mode $(\ell=0)$ rules out its previous classification as a RR Lyrae type star. The distribution of the oscillation power with respect to wavelength indicates a significant horizontal velocity component of oscillations characteristic of high radial order gravity modes which are predicted to be observed in a $\gamma$ Doradus type star.

The fact that low frequency gravity modes are present is crucial for the potential of asteroseismology of $\gamma$ Doradus stars. The research is very interesting in probing the stellar interior and particularly the core, since these modes propagate deep below the surface. In order to achieve this asteroseismological analysis, accurate period determination from long-time baseline observations is needed, consequently $\gamma$ Doradus stars are appropriate candidate targets for space missions such as COROT and KEPLER.

Acknowledgements. The authors thank the referee, Dr. A.B. Kaye, who provided many useful suggestions for improvements. We are also grateful to Dr. J. Tully for his careful reading of the manuscript.

\section{References}

Abt, H. A., \& Morrell, N. I. 1995, ApJS, 99, 135

Aerts, C. 1996, A\&A, 314, 115

Aerts, C., \& Krisciunas, K. 1996, MNRAS, 278, 877

Aerts, C., \& Kaye, A. B. 2001, ApJ, 553, 814

Aerts, C., Cuypers, J., De Cat, P., et al. 2004, A\&A, 415, 1079

Balona, L. A. 1987, MNRAS, 224, 41

Balona, L. A. 1998, ASP Conf. Ser., ed. P. A. Bradley, \& J. A. Guzik, 135, 120

Balona, L. A., Bohm, T., Foing, B. H., et al. 1996, MNRAS, 281, 1315

Breger, M., Handler, G., Garrido, R., et al. 1997, A\&A, 324, 566

Bruntt, H., Catala, C., Garrido, R., et al. 2002, A\&A, 389, 345

De Pauw, M., Aerts, C., \& Waelkens, C. 1993, A\&A, 280, 493

Dupret, M.-A., De Ridder, J., De Cat, P., et al. 2003, A\&A, 398, 677

Dupret, M.-A., Grigahcène, A., Garrido, R., Gabriel, M., \& Scuflaire, R. 2004, A\&A, 414, L17

ESA 1997, The Hipparcos and Tycho catalogues, ESA-SP 1200

Eyer, L. 1998, Ph.D. Thesis, Geneva Observatory, Switzerland

Fekel, F. C., Warner, P. B., \& Kaye, A. B. 2003, AJ, 125, 2196

Foster, G. 1995, AJ, 109, 1889

Grigahcène, A. 2004, Ph.D. Thesis, Universidad de Granada, Spain

Guzik, J. A., Kaye, A. B., Bradley, P. A., Cox, A. N., \& Neuforge, C. 2000, ApJ, 542, L57

Handler, G., Balona, L. A., Shobbrook, R. R., et al. 2002, MNRAS, 333, 262

Handler, G. 1999, MNRAS, 309, L19

Harmanec, P. 1999, A\&A, 341, 867

Hatzes, A. P. 1998, MNRAS, 299, 403

Henry, G. W., Fekel, F. C., \& Henry, S. M. 2005, AJ, 129, 2815

Kaye, A. B., Handler, G., Krisciunas, K., Poretti, E., \& Zerbi, F. M. 1999a, PASP, 111,840

Kaye, A. B., Henry, G. W., Fekel, F. C., \& Hall, D. S. 1999b, MNRAS, 308, 1081

Kazarovets, E. V., Samus, N. N., Durlevich, O. V., et al. 1999, IBVS 4659

Kennelly, E. J., Walker, G. A. H., \& Merryfield, W. J. 1992, ApJ, 400, L71

Kennelly, E. J., Walker, G. A. H., Catala, C., et al. 1996, A\&A, 313, 571

Krisciunas, K., Griffin, R. F., Guinan, E. F., Luedeke, K. D., \& McCook, G. P. 1995, MNRAS, 273, 662

Le Contel, J.-M., Mathias, P., Chapellier, E., \& Valtier, J.-C. 2001, A\&A, 380, 277

Mathias, P., Le Contel, J.-M., Chapellier, E., et al. 2004a, A\&A, 417, 189 (Paper I)

Mathias, P., Chapellier, E., Le Contel, J.-M., et al. 2004b, ESA SP-538, Second Eddington Workshop, 9-11 April 2003, Palermo, Italy, ed. F. Favata, S. Aigrain, \& A. Wilson, 355

Osaki, Y. 1971, PASJ, 23, 485

Poretti, E., Koen, C., Bossi, M., et al. 2002, A\&A, 384, 513

Royer, F., Grenier, S., Baylac, M.-O., Gómez, A. E., \& Zorec, J. 2002, A\&A, 393, 897

Vogt, S. S., \& Penrod, G. D. 1983, PASP, 95, 565

Zerbi, F. M., Rodríguez, E., Garrido, R., et al. 1997, MNRAS, 292, 43

Zerbi, F. M., Rodríguez, E., Garrido, R., et al. 1999, MNRAS, 303, 275 